Przemysław Wolski*, Paweł Szerszeń**

\title{
CHATBOTS UND VOICEBOTS AUF E-LEARNING- -PLATTFORMEN IN DER FÖRDERUNG DER BERUFSBEZOGENEN GESPRÄCHSKOMPETENZ IM DAF/DAZ UNTERRICHT
}

\section{CHATBOTS AND VOICEBOTS ON E-LEARNING PLATFORMS TO PROMOTE JOB-RELATED CONVERSATIONAL SKILLS IN DAF / DAZ LESSONS}

In der Studie wird die Frage behandelt, inwieweit interaktive Kommunikationssysteme zur Förderung der berufsbezogenen Gesprächskompetenz beitragen können, was durch ihre Implementierung in bereits funktionierende Lernplattformen beabsichtigt wird. Unter interaktiven Kommunikationssystemen werden hier sog. „sprechende Chatbots“ bzw. „Voicebots“ verstanden, deren Funktionsweise in der Analyse des Benutzerinputs und im Generieren von kommunikationsadäquaten Antworten mit Verwendung der maschinellen Sprachverarbeitung (natural language processing, NLP) und der künstlichen Intelligenz besteht. Im Rahmen des Aufsatzes wird zunächst versucht, die neuesten Entwicklungen im Bereich des e-learning-gestützten berufsbezogenen DaF/ DaZ-Unterrichts kritisch zu erfassen. Im Fokus des zweiten Teils des Beitrags stehen Elemente subjektiver Theorien von Studierenden, die in einer standarisierten Befragung erhoben wurden. Die sich auf den Einsatz interaktiver Systeme in universitären Deutschals-Fremdsprache-Kursen beziehenden Meinungen werden im Zusammenhang mit ausgewählten psychischen Dispositionen wie Kontrollüberzeugung, Machiavellismus oder Akzeptanz der konstruktivistischen Didaktik unter die Lupe genommen. Darauf aufbauend wird diskutiert, wie in derartigen Lernumgebungen der Prozess der Aufgabengestaltung aussehen könnte. Auf der Grundlage der konnektivistischen Lerntheorien wird

\footnotetext{
* Dr., Universität Warschau, Polen.

** Dr. habil., Universität Warschau, Polen.
} 
auch der Versuch unternommen, ein Modell der digital gestützten Aufgabenlösung im Bereich des Blended Learning vorzuschlagen.

Schlüsselwörter: E-Learning, Chatbot, Voicebot, DaF, berufsbezogener Sprachunterricht

The study addresses the question of the extent to which interactive communication systems can contribute to the promotion of job-related conversational competence, which is intended by their implementation in already functioning learning platforms. Interactive communication systems are understood here as so-called "talking chatbots" or "voicebots", which function by analyzing the user input and generating communicationadequate answers using natural language processing (NLP) and artificial intelligence. They usually consist of three parts: a database (the knowledge base) which provides a framework for the "intelligence" of the system, a software engine which controls the interface and the interpreter which communicates with the interface through its analyzer and generator. In the case of a talking chatbot, the interface is equipped with a speech recognition software and speech synthesis software. In the case of a chatbot, which complements an e-learning platform for the promotion of job-related linguisticcommunicative competence, the database consists of entries originating from the corpora of the spoken language or collected through procedures of language needs assessment.

The article first attempts to critically assess the latest developments in the field of e-learning supported vocational $\mathrm{DaF} / \mathrm{DaZ}$ teaching. The second part of the article focuses on elements of subjective theories of students that were collected in a standardized survey. The opinions concerning the use of interactive systems in university German as a foreign language courses will be examined in the context of selected psychological dispositions such as control conviction, Machiavellianism or acceptance of constructivist didactics.

Based on this, it is discussed how the process of task design could look like in such learning environments. On the basis of connectivist learning theories, an attempt is also made to propose a model of digitally supported task solving in the field of blended learning.

Keywords: e-learning, chatbot, voicebot, DaF, vocational language training

\section{Einleitung}

Eine rapide Entwicklung der Kommunikations- und Informationstechnologien (KIT) beeinflusst immer stärker unseren Alltag. Was noch vor einiger Zeit episodisch in Anspruch genommen wurde bzw. lediglich unserer Unterhaltung diente, prägt immer mehr unser tägliches Leben und erzeugt neue Gewohnheiten und Kommunikationspraktiken.

Elektronische bzw. digitale Medien halten seit einiger Zeit immer mehr Einzug in der Lern- und Arbeitswelt, wovon Ergebnisse mehrerer Studien zeu- 
gen, darunter der Trendstudie, mmb Learning Delphi 2016, einer Befragung von E-Learning-Expertinnen und -Experten zum digitalen Lernen in Betrieben im Jahr 2016, nach der die Sprach- o. Fremdsprachenkenntnisse zu den wichtigsten Themen/Inhalten des elektronischen bzw. digitalen Lernens in den kommenden drei Jahren gezählt werden. Eine zunehmende Bedeutung der o. g. Medien wurde vor kurzem auch im Schulwesen erkannt, worauf u. a. eine Studie der Wirtschaftsprüfungs- und Beratungsgesellschaft $\mathrm{PwC}$ aus dem Jahr 2018 hindeutet, nach der an vielen deutschen Schulen der Einsatz elektronischer Lehr- und Lernmaterialien bald der Normalfall sein könnte. Demnach soll der digitale Anteil an den Gesamterlösen im Schulbuchmarkt von derzeit 4,3 Prozent bis 2021 auf fast 40 Prozent steigen (vgl. PricewaterhouseCoopers 2019, online).

Die Glottodidaktik [Didaktik des (Fremd-)sprachenlehrens und -lernens], wie auch andere Didaktiken versucht seit langem das Potential von technischen Neuheiten in die sprachdidaktische Praxis zu implementieren, zuerst in Form sprachdidaktischer (geschlossener) Computerprogramme, gegenwärtig im Zuge der Entwicklung des Internets und immer leistungsstärkerer Computer/Laptops als Lernplattformen und linguistisch intelligente Lernsysteme, die immer wieder verbessert und um neue Funktionalitäten ergänzt werden. Eine der letztgenannten sind Chatbzw. Voicebots, die verschiedene Funktionen erfüllen und unter anderem zur Förderung der in diesem Beitrag befindlichen berufsbezogenen Gesprächskompetenz beitragen können.

In dieser Abhandlung wird die Frage diskutiert, inwieweit interaktive Kommunikationssysteme in Form von Chat- bzw. Voicebots zur Entwicklung der Gesprächskompetenz beisteuern können, was u. a. durch ihre Implementierung in funktionierende Lernplattformen beabsichtigt wird. Im Rahmen des Beitrags werden zuerst die gegenwärtigen Entwicklungen im Bereich der berufsbezogenen $\mathrm{DaF} / \mathrm{DaZ}$-Unterrichts in der Ära des digitalen Wandels kritisch erfasst. Im Vordergrund des zweiten Aufsatzteils stehen Elemente subjektiver Theorien von Studierenden, die in einer standarisierten Befragung erhoben wurden. Die sich auf den Einsatz interaktiver Systeme in universitären Deutsch-als-Fremdsprache-Kursen beziehenden Meinungen werden im Zusammenhang mit ausgewählten psychischen Dispositionen wie Kontrollüberzeugung, Machiavellismus oder Akzeptanz der konstruktivistischen Didaktik unter die Lupe genommen. Darauf knüpfend wird die Frage behandelt, wie in solchen Lernumgebungen der Prozess der Aufgabengestaltung aussehen könnte. Auf der Grundlage der konnektivistischen Lerntheorien wird auch der Versuch unternommen, ein Modell der digital gestützten Aufgabenlösung im Bereich des Blended Learning vorzuschlagen. 


\section{Theoretische Prämissen}

Wie oben angedeutet, spielen die Kommunikations- und Informationstechnologien im Bereich der Didaktik(en) eine immer bedeutendere Rolle, was mit einer fortwährend steigenden Anzahl von E-Learning-Produkten allgemein zu beobachten ist. Die letzteren treten gegenwärtig in Form von immer ausgereifteren Hardwarelösungen mit der Funktion des Generierens und Speicherns der didaktischen Stimuli ist, darunter u. a. immer weniger populären Desktop-Computern sowie tragbaren Laptops, Tablets oder Smartphones oder als Produkte, die sich v.a. auf die Organisation des E-Learnings beziehen. Die Gestaltung des elektronischen Lernens ist mit Hilfe der Lernplattformen möglich, die schon mehr einem Jahrzehnt im Einsatz sind und meistens als ein auf einem Server installiertes und im Internet verfügbares System verstanden werden ${ }^{1}$, das ein bestimmtes Computerprogramm oder evtl. bestimmte Computerprogramme mit einer Datenbank bzw. Datenbanken verbindet, die zwecks Realisierung didaktischer Lernziele errichtet wurde(n). Darüber hinaus bieten die Lernplattformen Organisations-, Kommunikations- und Kooperationsmöglichkeiten, um auf diese Weise ein Lernen zu ermöglichen (vgl. Würffel 2010). Im Einsatz sind gegenwärtig v. a. die in der Fachliteratur erwähnten LCMS (Learning Content Management Systeme), die die Funktionalitäten der LMS (Learning Management Systeme) und CMS (Content Management Systeme) vereinen bzw. VCS (Virtual Classroom Systeme). Unter den populärsten Lernplattformen (LMS) kann man sowohl Open-Source-Produkte nennen, wie etwa Moodle, Edmodo, OLAT, ILIAS sowie viele kommerzielle Lernplattformangebote, wie z. B. Blackboard, Angel, WebCT, Desire2Learn, Pearsona eCollege, Edvance360, Jenzabar e-Racer, SharePoint u. a. (vgl. LMS Evaluation 2018, Capterra²).

Einige davon, wie etwa Moodle oder ILIAS bilden eine Basis für ausgebaute E-Learning-Dienste innerhalb der Institutionen im (Hoch)schulwesen. Immer populärer werden in den letzten Jahren Lernplattformen mit Fokus Fachsprachendidaktik, darunter u. a. solche Projekte wie Jasne - Alles klar (2016), Tell me more Campus oder LISST/LISTiG, vgl. dazu u. a. Szerszeń 2014), die neue Wege des Fach(fremd)sprachenlernens eröffnen (mehr dazu im Kap. 3).

Um die Rolle der Lernplattformen und jeglicher elektronischer Lehr- und Lernmittel in der Sprachdidaktik zu bestimmen, sollte man zuerst den sprachdidaktischen Prozess in Erinnerung rufen. Im angesprochenen Prozess beeinflusst

${ }^{1}$ In Wirklichkeit wird der Name Lernplattform nicht selten auch für Anwendungen verwendet, die nur einen Teil der in der Definition erwähnten Funktionalitäten einer ausgereiften Lernplattform erfüllen (vgl. LMS, CMS, VCS).

${ }^{2}$ https://www.capterra.com/learning-management-system-software/\#infographic [Zugriff am 11.02.2020]. 
der Lehrende mit bestimmten didaktischen Mitteln und bestimmten didaktischen Methoden den Lernenden insofern, als er bestimmte Kompetenzen (darunter auch Gesprächskompetenzen) bei ihm entwickelt. Mit anderen Worten: Der Kommunikationsvorgang „Erlernen einer Fremdsprache“ besteht aus einzelnen Kommunikationsakten, deren Invarianten der Lernende, der Lehrende, der Text (im Fall der Gesprächskompetenz ${ }^{3}$ - der mündliche Text bzw. Äußerung) und das allgemeine Ziel der Kommunikationssituation sind. Die weiteren Elemente dieser Kommunikationssituationen: das Medium, die Sprachart, die Art und der Ort der Interaktion, die Zahl der Kommunikationspartner usw. sind in einem bestimmten Rahmen variabel und den drei Invarianten untergeordnet. Somit dienen Texte im Sprachunterricht als Impuls immerhin, durch den die Entwicklung von Sprachkompetenzen überhaupt möglich ist. In Bezug auf gesprochene Texte in der Sprachdidaktik kann man in Anlehnung an Grucza/ Szerszeń 2012 u. a. folgende Grundfragen formulieren: (1) Inwiefern können entsprechende Texteigenschaften die Internalisierung von GK beeinflussen? (2) Wie sollen didaktische Texte konstruiert werden, um einen beabsichtigten Stand der Internalisierung von GK bei den Lernenden zu erzielen? (3) Mit welchen Medien können didaktische Texte effektiv angewandt werden, um eine optimale Internalisierung von GK zu gewährleisten und welche Rolle spielen dabei LP mit Erweiterung um Chat- bzw. Voicebots (siehe die Interaktion auf Schema 1)? Diese und weitere Detailfragen (s. u.) stehen im Fokus der geplanten Forschungsmaßnahmen zur Entwicklung von Chatbots für die Zwecke des fachbezogenen DaF-Unterrichts.

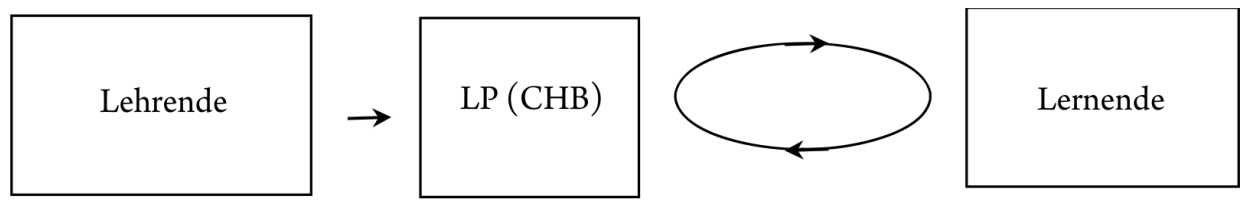

Schema 1. (eigene Darstellung)

In der Glottodidaktik werden schon seit langem zahlreiche Untersuchungen zur Erforschung der Entwicklung von Sprachkompetenzen betrieben. Nicht behandelt wurde die Frage, inwieweit interaktive Kommunikationssysteme zur Förderung der berufsbezogenen Gesprächskompetenz beitragen können, was u. a. durch ihre Implementierung in bereits funktionierende Lernplattformen beabsichtigt wird. Unter interaktiven Kommunikationssystemen werden hier sog. „sprechende Chatbots“ bzw. „Voicebots“ verstanden, deren Funktionsweise in der Analyse des Benutzerinputs und im Generieren von kommunikationsad-

${ }^{3}$ Im Weiteren abgekürzt als GK. 
äquaten Antworten mit Verwendung der maschinellen Sprachverarbeitung (natural language processing, NLP) und der künstlichen Intelligenz besteht.

Diese Systeme setzen sich in der Regel aus drei Teilen zusammen: einer Datenbank (der Wissensbasis) die einen Rahmen für die „Intelligenz" des Systems gewährleistet, einer Software-Engine, die die Schnittstelle steuert und dem Interpreter, der durch seinen Analyzer und Generator mit der Schnittstelle kommuniziert. Bei einem sprechenden Chatbot ist die Schnittstelle mit einer Spracherkennungssoftware und Sprachsynthese-Software ausgestattet. Im Fall eines Chatbots, der eine E-Learning-Plattform zur Förderung der berufsbezogenen sprachlich-kommunikativen Kompetenz ergänzt, besteht die Datenbank aus Einträgen, die aus den Korpora der gesprochenen Sprache stammen, bzw. durch Prozeduren der Sprachbedarfsermittlungen erhoben wurden.

Es muss übrigens betont werden, dass mündliche Interaktion als interaktive Aktivität in allen Curricula zum Fachfremdsprachenunterricht verankert wird. Es sollte auch nicht unerwähnt bleiben, dass diese Fertigkeit in der Entwicklung der fremdsprachlichen Handlungsfähigkeit im Beruf in zwei Formen auftritt: erstens in der einsprachigen mündlichen Interaktion, in der Sprachverwendende abwechselnd als Sprechende und Hörende handeln, zweitens in der mündlichen Sprachmittlung, bei der es dem Sprechenden nicht darum geht, seine eigenen Absichten zu realisieren, sondern darum, zwischen Gesprächspartnern zu vermitteln. Diese Tätigkeit bedarf also eines oder mehrerer Gesprächspartner, mit deren Hilfe auf der Basis des Prinzips der Kooperation, durch das Aushandeln von Bedeutungen das Gespräch entsteht (vgl. Demenko 2015, 17-18). Diese Aktivität wird in Ansätzen bereits auf dem Niveau A1 gefördert und lässt sich durch den Einsatz von Voicebots auf Lernplattformen simulieren.

Bei den sprachmittelnden Aktivitäten geht es dem Sprechenden nicht darum, seine eigenen Absichten zu realisieren, sondern darum, zwischen Gesprächspartnern zu vermitteln, die Sprecher verschiedener Sprachen sind. Nach Kiefer (2016, 78-79) sind es eben grenzüberschreitend agierende Unternehmen, bei denen diese Aktivität im Rahmen technischen Wissenstransfers eine relevante Aufgabe in der beruflichen Wirklichkeit ist. Demnächst ist die sprachliche Mittlertätigkeit bei der Erklärung von Verrichtungen, eine plastisch darstellbare Aufgabe im Rahmen einer Unterrichtseinheit oder eines Projekts. Somit ist zu schlussfolgern, dass sich auch diese Aktivität mit Verwendung der Chatbots simulieren ließe.

Der Begriff der Lerngelegenheit stammt aus der konstruktivistischen Fremdsprachendidaktik und nimmt an, dass die Lernenden das sie umgebende Sprachangebot selektiv und fokussiert wahrnehmen (vgl. Wolski 2017). Für eine solche Selektion und Fokussierung sind sowohl lernerexterne als auch -interne Einflussfaktoren verantwortlich. $\mathrm{Zu}$ den lernerexternen Faktoren zählt z. B. die Aufgabenstellung oder perzeptive Salienz (inwieweit die Aufgabe „auf sich aufmerksam 
macht"), die lernerinternen Faktoren sind u. a. Vorwissen, Sprach- und Sprachlernbewusstheit, Lernstile, oder subjektive Lernertheorien. Die in den Aufgaben „enthaltenen" semantischen und strukturellen Eigenschaften der Zielsprache werden, in verschiedenem Grad, mit den vorhandenen sicheren lernersprachlichen Wissensbeständen abgeglichen. Dies kann im Fall einer (subjektiv) wahrgenommenen Diskrepanz zur L2-Hypothesenbildung und zur Erhöhung der linguistischen Kompetenz (und damit der Handlungsfähigkeit im Beruf) führen.

\section{Umsetzung der Lernplattformen im berufsbezogenen FU Chat- und Voicebots}

Ein Teil von Lernplattformen findet Einsatz im fachbezogenen Fremdsprachenlernen. In Wirklichkeit funktionieren auf dem Markt viele solche Produkte, die viel versprechen, aber dafür bedeutend weniger leisten. Dies betrifft v. a. die versicherte Kompetenzentwicklung produktiver Art (Sprechen, Schreiben) wie auch stark eingeschränkte Interaktionsmöglichkeiten und reduzierten Feedbackumfang. Eine weitere wichtige Frage ist, ob und inwieweit die konkreten neuen E-Learning-Produkte (lediglich) das (Fach)Fremdsprachenlernen unterstützen oder gar neue Lernmethode darstellen sowie die daraus resultierende weitere Frage: inwieweit sie das bestehende Lernerwissen und die Lernerkompetenzen aktivieren oder ein neues Lernerwissen/neue Lernerkompetenzen erzeugen etc. (vgl. Grucza/Szerszeń 2012). Gemeint sind dabei adaptive bzw. adaptierbare Lernumgebungen oder gar linguistisch intelligente Lernsysteme (s. u. a. LISTiG, Jasne-Alles klar), die z. T. neue Wege des (Fach-)Fremdsprachenerwerbs eröffnen. Eine wichtige Ergänzung bzw. Erweiterung für die bestehenden Lösungen bieten auch solche Programme, wie etwa Tell me more Campus, die Chat- und Voicebots integrieren. In der Praxis (in Bezug auf TMM Campus) manifestiert sich die Rolle der Bots in einem virtuellen Gespräch zu ausgewählten Themen (z. B. Reiseorganisation). Im Fall der dieser Übungsart wird eine ungezwungene Unterhaltung am PC mit einem Muttersprachler (für amerikanisches Englisch) simuliert. Hierzu ist anzumerken, dass eine Konversation zwar möglich ist, aber erstens in einem begrenzten Rahmen (begrenzte Themenauswahl), zweitens nur gesteuert (d.h. oft auf die Wahl von mündlich vorgeschlagenen Varianten der Antwort reduziert) und drittens nur unter Beachtung vorgegebener Phrasen (Wortschatzes). Nichtsdestoweniger bietet die Lernplattform mit der Funktion „virtuelles Gespräch“ eine interessante Übungsart zur Verfügung, die erweiterungsfähig ist und besonders im fachfremdsprachlichen Kontext (v.a. bei der situationsbedingten Fachwortschatzverwendung) einsetzbar ist.

Lernziele und Lerninhalte (darunter auch Datenbanken der didaktischen Chatbots) werden immer unter Einbeziehung der zur Verfügung stehenden In- 
formationsquellen ermittelt. Methodisch gesehen, dominieren nach C. Efing (Efing 2016, 21) Ansätze, die eine Inhaltsanalyse von Ausbildungsverordnungen und Rahmenlehrplänen vornehmen, oder eine sprachliche Analyse von Lehrbüchern und anderem Textmaterial durchführen. Ergänzend werden andere empirische Verfahren eingesetzt, wie Interviews, Hospitationen und teilnehmende Beobachtung.

Infolge solcher Untersuchungen kann ein Korpus authentischer Sprachhandlungen gewonnen werden, das „zu verschiedensten Zwecken ausgewertet und aufbereitet werden kann“. Ein solches Korpus bildet z. B. die Grundlage der computergestützten Dialogsysteme, u. a. Chatbots (vgl. Demenko 2015, 27). Den nächsten Schritt der Planung bildet die Bestimmung der lexikalischen und grammatikalischen Lernpensen. Auf dieser Stufe kann auf die Ergebnisse der Fachsprachenforschung und der Fehleranalyse zurückgegriffen werden, mit besonderer Berücksichtigung der für die Zielgruppe und ihre Lernziele erfassten Textsorten. Im letzten Schritt werden die chatbotgestützen Aufgabenstellungen mit bestehenden E-Learning-Kursen integriert bzw. neue Kurse entwickelt.

\section{Forschungsprojekt „Subjektive Theorien über Chatbots in digitalgestützten Kursen Deutsch als Fremdsprache”}

Wie bereits erwähnt, werden schon seit langem zahlreiche Untersuchungen zur Erforschung der Entwicklung von fremdsprachlichen Sprachkompetenzen im digitalen Kontext betrieben, relativ selten sind Untersuchungen zum Einsatz interaktiver Kommunikationssysteme. Zu erwähnen sind:

- Studie zu Lernerhaltungen gegenüber der Kommunikation mit menschlichen Partnern und Chatbots (Gallagher/Thompson/Howarth 2018),

- Studie zum Interesse am chatbotgestützten Englischunterricht (Fryer 2018).

Der vorliegende Beitrag thematisiert behandelt Beziehungen zwischen subjektiven Theorien von erwachsenen Fremdsprachenlernern über den Einsatz von Chatbots und ihren ausgewählten Persönlichkeitsmerkmalen.

Die Population, auf die sich die beschriebene Untersuchung bezieht, sind polnische Studierende der Universität Warschau, die an berufsbezogenen, digitalgestützten Kursen Deutsch als Fremdsprache teilnehmen, in denen ergänzend die Lernplattform Moodle eingesetzt wird.

In der explorativen Phase des Projektes, die hier nicht behandelt wird, werden in einem mündlichen, offenen Interview studentische Teilnehmerinnen eines Seminars befragt, in dem chatbotgestützte Aufgaben gelöst werden. Ihre Aussagen konzentrieren sich auf erwartete Vorteile des Einsatzes von interaktiven Systemen, Erwartungen bezüglich des Gesprächsablaufs und bisherige Erfahrungen mit derartiger Software. 
Die systematische Stichprobe in der quantitativen Phase wird im Quotenverfahren erhoben, mit der Absicht, die Eigenschaften der Population möglichst genau wiederzugeben.

Vor allem wegen charakteristischer Eigenschaften der Institution (Universität) war eine Erhebung von Zufallsstichproben unmöglich. Die sog. freiwillige Stichprobenauswahl wird normalerweise bei Probanden mit hohem Bildungsniveau, aus höheren sozialen Schichten, mit hohem sozialen Status, höherem Intelligenzniveau, höherem Niveau der sozialen Anerkennung, sowie höherem Sozialisationsniveau eingesetzt (vgl. Wolski 2008).

Das Ziel der Untersuchung besteht darin, die Zusammenhänge zwischen bestimmten Meinungen über den Einsatz der Chatbots im E-Learning und einigen signifikanten Persönlichkeitsmerkmalen aufzudecken. Persönlichkeitsmerkmale und Einstellungen werden nicht direkt gemessen, sondern über ihren Einfluss auf subjektive Theorien der Befragten ermittelt. Der Schwerpunkt liegt in der Frage, ob Lernende, die eine positive Meinung der konstruktivistisch geprägten Fremdsprachendidaktik gegenüber vertreten, bei ihren Lernhandlungen selbst innovative Tools bevorzugen und ihnen positiven Einfluss auf den Fremdsprachenerwerb zuschreiben.

Es wird angenommen, dass Handlungsentscheidungen beim Fremdsprachenlernen sowohl aus unbewussten Persönlichkeitsmerkmalen und generalisierten Erfahrungen resultieren können als auch aus bewussten Überlegungen, die in Einstellungen und subjektiven Theorien münden. Demzufolge wurden insgesamt 4 abhängige Variablen ausgesondert: der Grad des Machiavellismus, der Grad der Kontrollüberzeugung, die Haltung zum Chatboteinsatz und das Niveau der Akzeptanz gegenüber der konstruktivistischen Fremdsprachendidaktik in den individuellen (subjektiven) Lerntheorien.

Der Grad des Machiavellismus ist ein Persönlichkeitsmerkmal, das als Bündel von Eigenschaften verstanden wird, die auf das Manipulieren anderer abzielen - was mit dem Negieren des Rechtes auf Autonomie gleichzusetzen ist. Für Personen mit einem hohen Grad des Machiavellismus ist leider eine hohe Effektivität im Erreichen von Zielen in interpersonalen Relationen charakteristisch.

Die genannten Eigenschaften sind vor allem:

- Die Projektion des eigenen Manipulationsdrangs auf die anderen, die sich darin äußert, dass Machiavellisten gerade den anderen die Lust am Manipulieren zuschreiben. Andererseits werden die Mitmenschen als manipulationswillig eingestuft und ihre Subjektivität wird ihnen aberkannt.

- Für das Erreichen von „positiven” Zielen werden Verstöße gegen moralische Normen zugelassen.

- Die ausgeprägte Rationalität in Kontakten verbindet sich mit dem Misstrauen und dem Missachten der Bedürfnisse der Mitmenschen.

- Die Machiavellisten verspüren einen ständigen Erfolgsdrang, sind zugleich emotional immun und kalt. 
Es wurde erwartet, dass die „Machiavellisten“ unter universitären Bedingungen u. a. folgende Feststellungen bestätigen werden:

- „Professoren haben das Recht, Studierende zu manipulieren“.

- "Manchmal betrüge ich, wenn ich dadurch eine bessere Note bekomme“.

- „Ich freue mich, wenn ich eine bessere Note bekomme als ein Kommilitone, der besser ist als ich".

- „Wenn mir Dozenten Vorwürfe machen, lässt mich das kalt“.

Die Feststellung „Die Professoren sollten den Studierenden ihre Sympathie zeigen" wird eher verneint.

Die zweite Variable bildet der Grad der Kontrollüberzeugung (locus of control) nach dem Konzept Julian Rotters (vgl. Wolski 2008). Darunter wird die Art und Weise verstanden, wie das Subjekt die Zusammenhänge zwischen Verhalten und Verstärkungen (Handlungen und Folgen) ansieht.

Wird die Handlungsfolge vom Subjekt als Ergebnis angesehen, das nicht gänzlich mit der Handlungsintention übereinstimmt, wird dieser Zustand in der europäischen Kultur als Zufall, Vorsehung oder Glück interpretiert bzw. als Ergebnis der Kontrolle anderer Macht habender Menschen oder einer unberechenbaren Komplexität äußerer Kräfte (Gefühl der äußeren Kontrolle). Stimmen die Resultate mit der eigenen Handlung bzw. eigenen Eigenschaften überein, haben wir es mit dem Gefühl der inneren Kontrolle zu tun.

Eine subjektive Überzeugung von der Kontrolle der Verstärkungen kann die verstärkenden Wirkungen der Handlungsergebnisse determinieren. Ist nämlich jemand überzeugt, dass er kaum Einfluss hat, welche Strafen bzw. Belohnungen ihm begegnen werden, wird er wenig motiviert sein, das eigene Handeln entsprechend zu steuern.

Die Erwartungen dem Zusammenhang Handeln - Ergebnis gegenüber resultieren aus generalisierten Erfahrungen bzw. aus Erwartungen, die für einen bestimmten Situationstyp spezifisch sind. Im Laufe der Persönlichkeitsentwicklung entwickelt sich das Subjekt durch verschiedene, sich wiederholende Erfahrungen. In verschiedenen Situationen werden generalisierte Erwartungen den kausalen Zusammenhängen zwischen dem Verhalten und seinen Konsequenzen gegenüber gespeichert. In einer neuen Situation, die sich nicht eindeutig als schicksals- bzw. fertigkeitsbezogen zuordnen lässt, richtet sich der Mensch nach diesen Erwartungen. In bekannten Situationen, wie auch bei zufallsbedingten und fertigkeitsbezogenen Entscheidungen handelt das Subjekt nach anderen, situationsspezifischen Erwartungen.

Eine beständige und generalisierte Erwartung dem locus of control gegenüber ist eine individuelle Eigenschaft jedes Menschen und kann als eine Persönlichkeitsdimension behandelt werden. Sie bildet ein Kontinuum, das vom generalisierten Gefühl der äußeren Kontrolle (external locus of control) zum generalisierten Gefühl der inneren Kontrolle (internal locus of control) verläuft. 
Für den äußeren locus of control gilt die Feststellung:

„Wenn ich einen guten Lehrer habe, lerne ich bestimmt eine Fremdsprache“.

Den anderen Pol würde dann z. B. die Aussage markieren:

„Wenn ich mir Mühe gebe, finde ich bestimmt eine gute Stelle“.

Die dritte analysierte Variable ist das Niveau der Akzeptanz gegenüber der konstruktivistischen Fremdsprachendidaktik in den individuellen (subjektiven) Lerntheorien.

In den gegenwärtigen Bildungsansätzen wird die Rolle der affektiven Sphäre für den Lernerfolg betont. Die Lernerzentriertheit setzt ein gutes Selbstgefühl, positive Einstellungen zu den auszuführenden Aufgaben und ein Erfolgsgefühl der Lernenden voraus. Die Lehrenden werden nicht mehr mit der äußeren Steuerung der Lernprozesse beauftragt, sie fungieren eher als Helfer, Berater und classroom manager und sind vor allem für die Bewusstmachung der Prozesse und Inhalte zuständig, die in einem konkreten Lernakt einzusetzen sind.

Im konstruktivistischen Sinne besteht der Unterricht im gemeinsamen Handeln der Lernenden und der Lehrpersonen, das zum Ziel hat, Mittel und Wege zu erarbeiten, die die beiden Seiten in die Lage versetzen, immer autonomer Entscheidungen über Lerninhalte zu treffen, das Lernen zu planen und $\mathrm{zu}$ evaluieren.

Die in der Befragung enthaltenen Fragen betrafen die Auswertung von Situationen, in denen die erwähnten Funktionen der Lehrpersonen von Bedeutung waren. Positiv werden u. a. Feststellungen bewertet, wie:

„Die Lernenden sollten selbst entscheiden, welchen Wortschatz sie brauchen“.

„Die Lernenden sollten wissen, wozu die von ihnen gemachten Übungen dienen“.

Abgelehnt wird dagegen z. B. die Feststellung „Es ist nicht gut, wenn die Klasse Gruppenarbeit macht. Da kann der Lehrer nichts kontrollieren“.

Die vierte Variable bildet die Haltung zum Chatboteinsatz (als Bündel bestimmter Einstellungen verstanden), die in den subjektiven Lerntheorien manifestiert wird.

Die untersuchten Variablen sind nicht beobachtbar. Die Informationen darüber werden über Indikatoren ermittelt. Als Indikatoren fungieren in der explorativen Phase die Aussagen während der Interviews, in der eigentlichen Befragung sind es Antworten auf die Fragen des Fragebogens. Dem Wert der unabhängigen Variablen werden Zahlen (1-5) zugeordnet, was ihre statistische Analyse ermöglicht. Eine Zahl entspricht also dem Wert eines Faktors, der die Wahl einer konkreten Antwort verursacht. 
Die Pilotuntersuchung, als Überprüfung des Untersuchungswerkzeugs gedacht, wurde mit einer Stichprobe von ca. 30 Personen durchgeführt. Die eigentliche Untersuchung umfasste ca. 200 Personen. Das Werkzeug ist ein Fragebogen in der Muttersprache der Befragten (Polnisch) mit einer geschlossenen Anzahl von möglichen disjunktiven Antworten (bei jeder Frage war nur eine Antwort möglich), der online ausgefüllt wird.

Der Fragebogen besteht aus 4 Segmenten, die den untersuchten Variablen entsprechen:

- Machiavellismus (5 Fragen),

- locus of control (5 Fragen),

- konstruktivistische Fremdsprachendidaktik (10 Fragen),

- Einstellung zur Arbeit mit Chatbots (5 Fragen).

Die anderen Fragen beziehen sich auf das Geschlecht, die Ausbildung der Eltern und die Anzahl der gelernten Fremdsprachen. Die Untersuchung wurde im Juni 2019 durchgeführt und erbrachte die unten angeführten Ergebnisse.

Variablen (Mittelwert):

- Grad des Machiavellismus (2,64),

- innere vs. äußere Kontrollüberzeugung $(3,20)$,

- Akzeptanz der konstruktivistischen Didaktik im Unterricht DaF $(3,76)$,

- Haltung zum Chatboteinsatz $(3,57)$.

Es wurde eine schwache Korrelation zwischen der Akzeptanz der konstruktivistischen Didaktik und der positiven Haltung zum Chatboteinsatz festgestellt $(0,35)$, was von einer geringen aber definitiven Beziehung zeugt. Bei allen anderen Variablen wurden keine Korrelationen entdeckt.

Bei den einzelnen Segmenten konnten 4 Hauptprofile ermittelt werden, die Antworten mit dem Wert über 3,0 enthalten („Machiavellisten“, „Selbstbewusste“, „Konstruktivisten“ und „Chatbot-Anhänger").

Der Vergleich der Antworten der Hauptprofile mit dem Mittelwert der Antworten der Gesamtprobe ergab folgende Werte:

- „Machiavellisten“ vs. „Selbstbewusste“ 3,24; Mittelwert für die Gesamtprobe: 3,20; vs. „Konstruktivisten“ 3,72 (Gesamtprobe 3,76); vs. „Chatbot-Anhänger" 3,57 (3,57),

- „Selbstbewusste“ vs. „Konstruktivisten“ 3,82; Mittelwert für die Gesamtprobe 3,76; vs. „Chatbot-Anhänger“ 3,60 (3,57),

- „Konstruktivisten“ vs. „Selbstbewusste“ 3,86 (3,20),

- "Chatbot-Anhänger“ vs. „Machiavellisten“ 2,70 (2,64); „Selbstbewusste“ 3,22 (3,20); vs. "Konstruktivisten” 3,89 (3,76).

Aus diesen Ergebnissen kann geschlossen werden, dass Personen mit einem stark generalisierten Gefühl der inneren Kontrolle auch Anhänger der konstruktivistischen Didaktik sind, und umgekehrt, die „Konstruktivisten“ ein generalisiertes Gefühlt der inneren Kontrolle haben (eher autonom sind). Für Personen, 
die den Einsatz von Voice- bzw. Chatbots positiv einschätzen, ist eine hohe Akzeptanz für die konstruktivistische Didaktik charakteristisch.

Das letzte Segment der Umfrage erhielt 5 Fragen zu Erwartungen zum Voiceboteinsatz (in Klammern der Mittelwert):

- Lernplattformen sollten Chatbots enthalten $(3,61)$,

- Voicebots beeinflussen positiv die Sprechfertigkeit $(3,67)$,

- Studierende, die Chatbots verwenden, machen weniger Fehler $(3,25)$,

- Studierende, die Chatbots verwenden, haben einen reicheren Wortschatz $(3,71)$,

- Studierende, die Chatbots verwenden, haben eine bessere Aussprache $(3,6)$.

\section{Ausblick}

Wie bereits angedeutet, hat das präsentierte Forschungsprojekt auch einen angewandten Aspekt, bei dem es sich um Entwicklung digitaler Lernplattformen handelt, die integrierte Voicebots enthalten. Die Grundlage dieser Kurse bilden im Rahmen der Germanistischen Institutspartnerschaft zwischen den Universitäten Wuppertal und Warschau erarbeiten Spezialkorpora mit authentischen Sprachhandlungen aus den Bereichen Callcenter, Pflege und Büro. Dieser Planungsschritt wird mit der Bestimmung lexikalischer und grammatikalischer Lernpensen initiiert. Auf dieser Stufe kann auch auf die Ergebnisse der Fachsprachenforschung und der Fehleranalyse zurückgegriffen werden, mit besonderer Berücksichtigung der für die Zielgruppe und ihre Lernziele erfassten Textsorten.

Für Lernziele Präsentieren, Verhandeln und Gesprächsführung, die in Lehrplänen am häufigsten vertreten sind (vgl. Weber 2018), können spezielle Übungssequenzen entwickelt werden, die $\mathrm{zu}$ einem Kommunikationstraining in der Fremdsprache führen. Die Aufgabengestaltung wird sich an typischen Prinzipien der Aufgabenstellung in Online-Phasen von E-Learning-Kursen orientieren. Es ist daher von Bedeutung, dass:

- die Aufgaben im kleinschrittigen Vorgehen gestaltet werden,

- die Arbeitsaufträge interessenorientiert sind,

- die Lernprodukte sowohl geschrieben als auch mündlich sind (der Voicebot vermittelt Informationen, die schriftlich zu fixieren sind und die Lernenden produzieren mündliche Aussagen, die vom Voicebot ausgewertet werden),

- es ist ein Raum zur Binnendifferenzierung vorhanden, es werden z. B. auch teilweise korrekte Aussagen akzeptiert,

- Aufgaben werden in verschiedenen Sozialformen gelöst, der Voicebot kann z. B. zwei Sprecher simulieren,

- Kommunikation und Austausch von Informationen werden gefördert, Aufgaben enthalten eine Informationslücke (information gap), 
- die Kreativität der Lernenden wird gefördert, z. B. reagiert das System positiv auf Aussagen, die nicht in der Datenbank vorhanden sind (vgl. Meister/ Shalaby 2014, 22; Wolski 2019, 141).

Dabei ist es wichtig, dass die Übungen an den entsprechenden Diskurs in der Muttersprache anknüpfen und auf das professionelle sprachliche Handeln in der Zielsprache vorbereiten. Die zu diesem Zweck dienenden Chatbots erfüllen eine ähnliche Rolle wie Gesprächspartner in kommunikativen Aufgaben im Klassenraum.

\section{Literatur}

Arich-Gerz, Bruno/Efing, Christian/Kiefer, Karl-Hubert (Hg.) (2016): Berufsbezogene und (inter)kulturelle Kompetenzen im deutsch-polnischen Kultur- und Wirtschaftsraum. Wuppertal: Roland Reischl Verlag.

Demenko, Grażyna (2015): Korpusowe badania języka mówionego. Warszawa: Akademicka Oficyna Wydawnicza EXIT.

Efing, Christian (2016): Sprachbedarfsermittlung - Curriculumsentwicklung - Aufgabenkonzeption. In: Arich-Gerz, Bruno/Efing, Christian/Kiefer, Karl-Hubert (Hg.): Berufsbezogene und (inter)kulturelle Kompetenzen im deutsch-polnischen Kulturund Wirtschaftsraum. Wuppertal, S. 13-29.

Efing, Christian/Kiefer, Karl-Hubert (Hg.), (2018): Sprache und Kommunikation in der beruflichen Aus- und Weiterbildung. Tübingen: Narr Francke Attempto.

Fryer, Luke (2018): Quantitative Methodology: Experimental and longitudinal advances in language learning research. In: Phakiti, Aek/De Costa, Peter/Plonsky, Luke/Starfield, Sue (Hg.): The Palgrave handbook of applied linguistics research methodology. London: Palgrave Macmillan, S. 55-77.

Gallacher, Andrew/Thompson, Andrew/Howarth, Mark (2018): "My robot is an idiot!" - Students' perceptions of AI in the L2 classroom. In: Taalas, Peppi/Jalkanen, Juha/Bradley, Linda/Thouësny, Sylvie (Hg.) Future-proof CALL: language learning as exploration and encounters - short papers from EUROCALL 2018, Research-publishing.net, S. 70-76, https://files.eric.ed.gov/fulltext/ED590612.pdf [13.02.2020].

Grucza, Sambor/Szerszeń, Paweł (2012): Potencjał dydaktyczny platform i programów e-learningowych. In: Studia Niemcoznawcze. XLIX, S. 611-628.

JASNE-ALLES KLAR! Mehrsprachig handeln mit Erfolg. Das Projekt im Rahmen des Lifelong Learning Programms der Europäischen Kommission (2016): http:// www.jasne.org [Zugriff am:13.02.2020].

Kiefer, Karl-Hubert (2016): Verlagerung von Produktionslinien eines deutschen Messgeräteherstellers nach Polen. In: Arich-Gerz, Bruno/Efing, Christian/Kiefer, Karl-Hubert (Hg.): Berufsbezogene und (inter)kulturelle Kompetenzen im deutsch-polnischen Kultur- und Wirtschaftsraum. Wuppertal. S. 63-79.

Meister, Hildegard/Shalaby, Dalia (2014): E-Learning. Handbuch für den Fremdsprachenunterricht. München: Hueber Verlag. 
PricewaterhouseCoopers (Hg.) (2019): Digitale Bildung: E-Books an Schulen vor dem Durchbruch. https://www.pwc.de/digitalebildung [Zugriff am:13.02.2020].

Szerszeń, Paweł (2010): Glottodydaktyka a (hiper)teksty internetowe, Warszawa: KJS.

Szerszeń, Paweł (2014): Aktuelle Tendenzen im computerunterstützten (Fach)Fremdsprachenunterricht. In: Zeitschrift für Interkulturellen Fremdsprachenunterricht. 1, S. 250-260, http://zif.spz.tu-darmstadt.de/jg-19-1/beitrag/Szerszen.pdf [13.02.2020].

Weber, Peter (2018): Förderung von Gesprächskompetenz im Bereich der beruflichen Bildung. In: Efing, Christian/Kiefer, Karl-Hubert (2018): Sprache und Kommunikation in der beruflichen Aus- und Weiterbildung. Tübingen: Narr Francke, S. 275-284.

Wolski, Przemysław (2008): Autonomia a konstruktywizm. In: Pawlak, Mirosław (Hg.) Autonomia w nauce języka obcego - co osiągnęliśmy i dokąd zmierzamy? Poznań, Kalisz, Konin: Wydawnictwo PWSZ w Koninie, S. 65-73.

Wolski, Przemysław (2017): Sprechende Chatbots im Fach(fremd)sprachenunterricht und im berufsbezogenen Fremdsprachenunterricht. In: Lingwistyka Stosowana. 22: 2, S. 138-148, http://alp.uw.edu.pl/wp-content/uploads/sites/315/2018/10/ 9-LS-22-P.-Wolski-Sprechende-Chatbots-im-Fachfremdsprachenunterricht.pdf [13.02.2020].

Wolski, Przemysław (2019): Förderung der berufsbezogenen Gesprächskompetenz durch interaktive Kommunikationssysteme - theoretische Grundlagen und praktische Anwendungen. In: Sprache im Beruf. 2, S. 133-141.

Würffel, Nicola (2010): DaF-/DaZ-Lernen in elektronischen Umgebungen. In: Krumm, Hans-Jürgen/Fandrych, Christian/Hufeisen, Britta/Riemer, Claudia (Hrsg.), Deutsch als Fremd- und Zweitsprache. Ein internationales Handbuch. Berlin, New York: Mouton de Gruyter (= Handbücher zur Sprach- und Kommunikationswissenschaft Bd. 35/1), S. 1227-1242. 